Article

\title{
Enhancement of High-Order Harmonic Generation due to the Large Gradient of the Electric Field Amplitude
}

\author{
Meiyan Qin, Yi Zeng, Xin Zeng and Qing Liao* \\ Hubei Key Laboratory of Optical Information and Pattern Recognition, Wuhan Institute of Technology, \\ Wuhan 430205, China; qinmeiyan@hust.edu.cn (M.Q.); 5706185352qq@gmail.com (Y.Z.); \\ m13545641674@163.com (X.Z.) \\ * Correspondence: liaoqing@wit.edu.cn
}

Received: 11 December 2018; Accepted: 10 January 2019; Published: 14 January 2019

\begin{abstract}
The influence of the waveform of circularly polarized laser field on high-order harmonic $(\mathrm{HH})$ generation from atoms is investigated by solving the time-dependent Schrödinger equation (TDSE) and by classical trajectory analysis, without assuming an initial transverse velocity. Both the $\mathrm{HH}$ simulation and the classical trajectory calculation demonstrate that the positive temporal gradient of the electric field amplitude is a key factor that makes the electron return to the parent ion possible. Moreover, the larger the temporal gradient of the field amplitude is, the more the electron trajectories will revisit the parent ion. Correspondingly, the enhancement of $\mathrm{HH}$ is observed. This is confirmed by the pulse-duration dependence of the harmonic yield driven by a circularly polarized laser field.
\end{abstract}

Keywords: circularly polarized laser pulse; strong field physics; high-order harmonic generation; pulse duration dependence of the harmonic yield

\section{Introduction}

The rapid development of attosecond physics, including the subject of high-order harmonic generation [1-5], ionization [6-10], target excitation [11-13], etc., has allowed us to probe ultrafast dynamics in atoms and molecules with unprecedented temporal and spatial resolutions. Recently, high-order harmonic $(\mathrm{HH})$ generation driven by circularly polarized laser fields has been a hot topic due to its potential application in the generation of circularly polarized attosecond pulses [14-17], which is a new tool for controlling and imaging ultrafast dynamics, such as charge transfer and migration and the generation of attosecond quantum electron currents for ultrafast magneto-optics.

A well-established mechanism for HH generation is semiclassical recollision [18,19], where the $\mathrm{HH}$ generation process consists of three steps: firstly an atom or molecule emits its electron to a continuous state; secondly the electron is accelerated in the laser field; and thirdly the accelerated electron recombines with the parent ion and a high energy photon is radiated, which is called HHs. Both experimental and theoretical investigations show that the harmonic efficiency is very sensitive to the polarization of the driving pulse [20-26]. In other words, the harmonic emission driven by a circularly polarized laser field is significantly suppressed compared with that driven by a linearly polarized laser field. The underlying mechanism is only those electrons which can return to the parent ion core are responsible for $\mathrm{HH}$ generation. However, in a circularly polarized field, the ionized electrons are transversely accelerated by the driving field, consequently missing the core [27-30]. In previous investigations, it has been shown that an initial transverse velocity can compensate the transverse drift induced by the circularly polarized laser field. Hence the electron trajectories with 
an initial transvers velocity can lead to the electron return and HH generation [27-30]. Whereas, the discussion on the factors leading to the electron return and $\mathrm{HH}$ generation driven by circularly polarized laser pulse is incomplete.

In this paper, we study the influence of the waveform of driving field on high-order harmonic $(\mathrm{HH})$ generation from atoms driven by circularly polarized laser pulses. It is found that the electron trajectories without an initial transverse velocity can also revisit the parent ion. Both the time-dependent Schrödinger equation (TDSE) simulation and the classical trajectory analysis show that the positive temporal gradient of the electric field amplitude is a key factor that makes the electron return to the parent ion possible. Moreover, the larger temporal gradient of the field amplitude is, the more electron trajectories can revisit the parent ion. Correspondingly, the harmonic yield is enhanced. This is confirmed by the pulse-duration dependence of the harmonic yield driven by a circularly polarized laser field. The classical trajectory analysis also shows that the maximum kinetic energy of the return electron, which agrees well with the harmonic cutoff position, is generally gained after multiple revisits. Our results provide a new way of controlling the $\mathrm{HH}$ emission in a circularly polarized laser field.

\section{Theoretical Model}

In this work, we investigate the HH generation from atom in a circularly polarized laser field by numerically solving a two-dimensional single-active-electron (SAE) TDSE (atomic units are used throughout this paper unless otherwise stated):

$$
i \frac{\partial}{\partial t} \Psi(r, t)=\hat{H}(r, t) \Psi(r, t) .
$$

The Hamiltonian reads

$$
\hat{H}(\boldsymbol{r}, t)=-\frac{1}{2} \nabla^{2}+V(\boldsymbol{r})+\boldsymbol{r} \cdot \boldsymbol{E}(t) .
$$

$\boldsymbol{E}(\mathrm{t})$ is the electric field and $V(r)$ is the model potential of the target. Here we use the SAE soft-core potential for atom, which is given by:

$$
V(\boldsymbol{r})=-\frac{1}{\sqrt{r^{2}+a^{2}}}
$$

with $a$ being the soft-core parameter. The TDSE is solved using the split-operator method [31]. The initial state is obtained by the imaginary-time propagation. Then the time-dependent wavefunction is obtained by real-time propagation, starting from this initial state. According to the Ehrenfest theorem [32], the time-dependent dipole acceleration $\ddot{d}(t)$ is given by:

$$
\ddot{d}(t)=-\Psi(\boldsymbol{r}, t)|\nabla V(\boldsymbol{r})+\boldsymbol{E}(t)| \Psi(\boldsymbol{r}, t) .
$$

After obtaining the dipole acceleration, the harmonic spectrum is given by the Fourier transform of $\ddot{\boldsymbol{d}}(t)$,

$$
\begin{gathered}
E_{X U V}=\int \ddot{\boldsymbol{d}}(t) \exp \left(-i q \omega_{0} t\right) d t \\
S_{q}=\left|E_{X U V}\right|^{2},
\end{gathered}
$$

where $q$ corresponds to the harmonic order.

The integrated harmonic yield between $\omega_{1}$ and $\omega_{2}$ is given by:

$$
\Delta \mathrm{I}=\frac{1}{\left(\omega_{2}-\omega_{1}\right)} \int_{\omega_{1}}^{\omega_{2}} S(\omega) d \omega
$$


The classical electron trajectory is calculated by solving the electron's Newton equation in the laser field [18,19]:

$$
\frac{d^{2} r(t)}{d t^{2}}=-E(t)
$$

The initial condition is given by $\boldsymbol{r}_{0}=\boldsymbol{v}_{0}=0$ at the time of tunneling.

\section{Numerical Results and Discussion}

We take the $\mathrm{He}$ atom as an example to investigate the $\mathrm{HH}$ generation from atom in a circularly polarized laser field. Hence, the soft-core parameter $a$ takes the value of 0.27 to simulate the ionization potential of the He atom $(24.26 \mathrm{eV})$. For a circularly polarized laser field propagating along the $\mathrm{z}$ axis, the $\mathrm{x}$ and $\mathrm{y}$ components of the electric field are respectively given by:

$$
\begin{aligned}
& E_{x}(t)=E_{0} f(t) \sin (\omega t), \\
& E_{y}(t)=E_{0} f(t) \cos (\omega t)
\end{aligned}
$$

Here, $E_{0}=\sqrt{\frac{2 I_{0}}{c \varepsilon_{0}}}$ is the field amplitude corresponding to the intensity $I_{0}, f(t)$ is the envelope of the laser field. When $f(t)=1$, the circularly polarized field has a constant amplitude. In Figure 1, the harmonic spectra for a circularly polarized field with a constant amplitude (panel a) and a sine-squared envelope (panel $b$ ) are presented. Three different wavelengths are considered, while the laser intensity is fixed at $1.0 \times 10^{14} \mathrm{~W} / \mathrm{cm}^{2}$. The total interaction time is 10 optical cycles for all the cases.

Interestingly, the harmonic spectra generated by the laser field with a constant amplitude and a sine-squared envelope possess quite different spectral structures. In the case of constant amplitude shown in Figure 1a, significant signals can only be observed below and near the ionization energy, as denoted by the black dash-dotted line. After that, the spectral intensity decreases very quickly. Moreover, the harmonic spectrum does not change too much when varying the wavelength of the driving laser. These features are quite different from those observed in the harmonic spectrum driven by a linearly polarized field, i.e. there is a plateau followed by a wavelength-dependent cutoff. The plateau and the dependence of the cutoff on the wavelength of the driving pulse are the typical features caused by the recollision mechanism. Hence, in the case of the laser field with constant amplitude, the harmonics caused by the recollision mechanism are significantly suppressed. They can't be observed in the spectrum. The significant suppression of the high-order harmonics implies that there is no sufficient returns of the electron. Whereas, in the case of laser field with a sine-squared envelope shown in Figure 1b, the HH spectra present the typical features caused by the recollision mechanism. In detail, there is an obvious plateau followed by a cutoff in each harmonic spectrum. The harmonic cutoff is extended when increasing the wavelength of the driving field. They are around $37.19 \mathrm{eV}, 52.03 \mathrm{eV}$, and $72.76 \mathrm{eV}$ for the wavelength of $800 \mathrm{~nm}, 1200 \mathrm{~nm}$, and $1600 \mathrm{~nm}$, respectively. If the ponderomotive energy of a free electron in a circularly polarized laser field is given by $U_{p}=\frac{\left(E_{0}\right)^{2}}{4 \omega^{2}}$, then the cutoff position satisfies $E_{\text {cutoff }} \cong I_{p}+2 U_{p}$, which is consistent with previous reports. The above harmonic spectral features indicate that sufficient electron recollision occurs to induce high-order harmonic generation in the case of sine-squared laser field. 

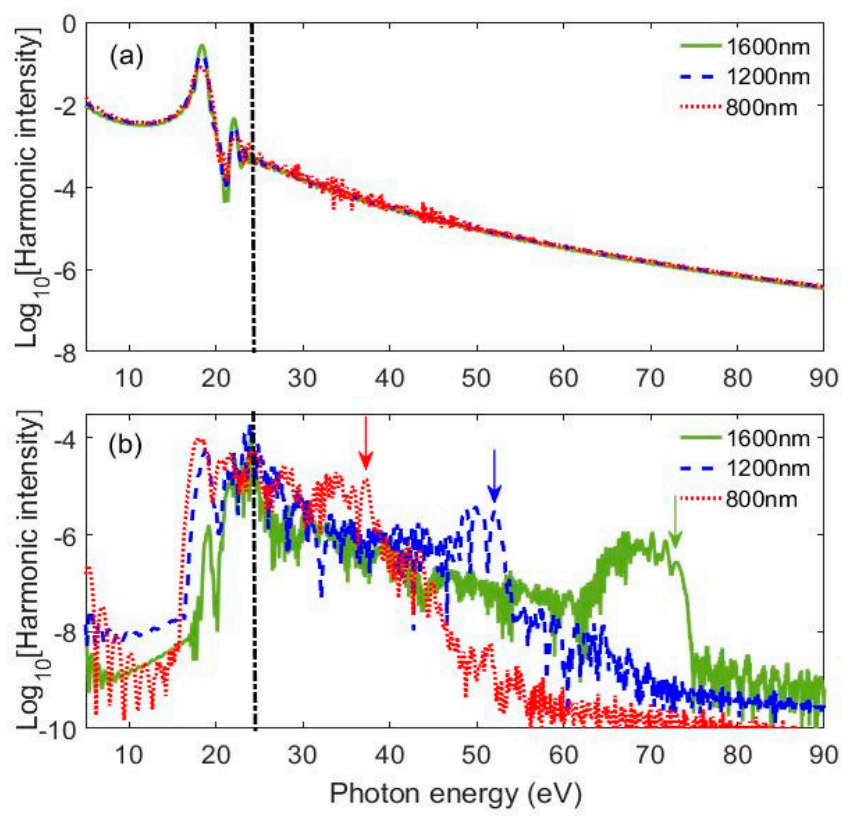

Figure 1. High-order harmonic spectra generated by a laser field with a constant amplitude (a) and with a sine-squared envelope $(\mathbf{b})$. Three different wavelengths are considered. The interaction time between He atom and the laser field is 10 optical cycles and the laser intensity is $1.0 \times 10^{14} \mathrm{~W} / \mathrm{cm}^{2}$.

To explore the universality of the above phenomenon, various envelopes and laser intensities for the driving field are considered. The results are presented in Figure $2 \mathrm{a}-\mathrm{d}$ for the cases of a constant amplitude (panel a), a trapezoidal envelope (panel b), a gaussian envelope (panel c), and a sine-squared envelope (panel d), respectively. Here, we consider two different pulse intensities of $2.0 \times 10^{14} \mathrm{~W} / \mathrm{cm}^{2}$ and $1.0 \times 10^{14} \mathrm{~W} / \mathrm{cm}^{2}$. The wavelength of the driving field is fixed at $800 \mathrm{~nm}$. The total interaction time is 10 optical cycles for all the cases, where the rising and falling time of the trapezoidal pulse is two optical cycles. As shown in these figures, a similar phenomenon to that in Figure 1 is observed. Namely, in the case of constant amplitude shown in Figure 2a, there are no significant signals above the ionization energy and the spectrum is not influenced significantly by varying the laser intensity. Whereas, when the field is added a finite envelope, the typical features of the harmonic spectrum appear. For each harmonic spectrum shown in Figure $2 b-d$, there is a plateau above the ionization energy and followed by a cutoff. The cutoff positions are almost the same for the three different envelopes when the intensities of the driving pulses are the same, as indicated by the red dashed and blue solid arrows in Figure $2 \mathrm{~b}-\mathrm{d}$. But the cutoff position is extended when increasing the pulse intensity. Moreover, the cutoff position satisfies $E_{\text {cutoff }} \cong I_{p}+2 U_{p}$ for all the cases. Hence, the high-order harmonics caused by the recollision mechanism are significantly suppressed, unless the driving field possesses an envelope. It is indicated that the time-varying amplitude of the driving field plays an important role in the electron return to the parent ion. 


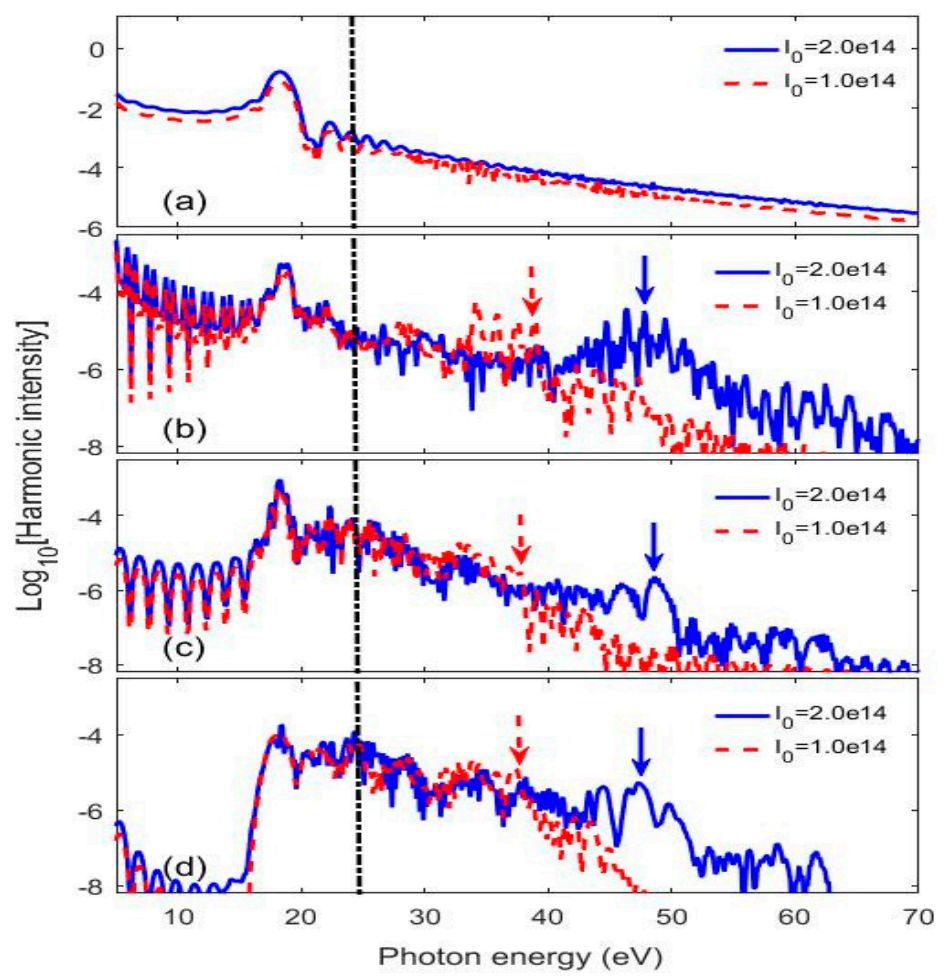

Figure 2. High-order harmonic spectra generated by a laser field with a constant amplitude (a), trapezoidal (b), gaussian (c), and sine-squared (d) envelopes. Two different intensities of the laser field $\left(2.0 \times 10^{14} \mathrm{~W} / \mathrm{cm}^{2}\right.$ and $\left.1.0 \times 10^{14} \mathrm{~W} / \mathrm{cm}^{2}\right)$ are considered. Other parameters are the same as used in Figure 1.

To explain the above finding, we perform an analysis of the classical trajectory of the electron in circularly polarized laser fields. As examples, the laser fields with constant amplitude and a sine-squared envelope are considered. The intensity, wavelength, and pulse duration of the two driving laser field are respectively $1.0 \times 10^{14} \mathrm{~W} / \mathrm{cm}^{2}, 800 \mathrm{~nm}$, and 10 optical cycles. The corresponding harmonic spectra are presented by the red dotted curves in Figure 1. By solving the Newton's Equation of the freed electron in the two driving laser fields, the classical trajectories of the electron are obtained. For both two laser fields, the electron is set to start from the origin at zero velocity $[18,19]$. The electron return is determined by the distance between the electron and the parent ion $r$. When $r$ is less than 4 a.u., then we treat this situation as an efficient return to the parent ion [33]. In Figures 3 and 4, we present a typical trajectory for the laser field with constant amplitude (Figure 3a-c) and four typical trajectories for the sine-squared laser field (Figure $3 \mathrm{~d}-\mathrm{i}$ ) and Figure $4 \mathrm{a}-\mathrm{f}$ ). We denote the four typical trajectories of sine-squared laser field as trajectory "a" (Figure 3d-f)), trajectory "b" (Figure 3g-i), trajectory " $\mathrm{c}$ " (Figure $4 \mathrm{a}-\mathrm{c}$ ), and trajectory " $\mathrm{d}$ " (Figure $4 \mathrm{~d}-\mathrm{f}$ ), respectively. The time-dependent $r$, the two-dimensional displacement of the electron, and the evolution of the electron kinetic energy for each trajectory are presented in the first, second, and third columns of Figures 3 and 4, respectively. In Figure $3 a, d, g$, and Figure $4 a, d$, the $x$ component of the electric field is presented by the pink dash-dotted curve. In addition, the electric field and the vector potential at the initial time for the four typical trajectories of sine-squared laser field are also depicted by the corresponding characters in Figure $4 \mathrm{~g}$,h, respectively. 

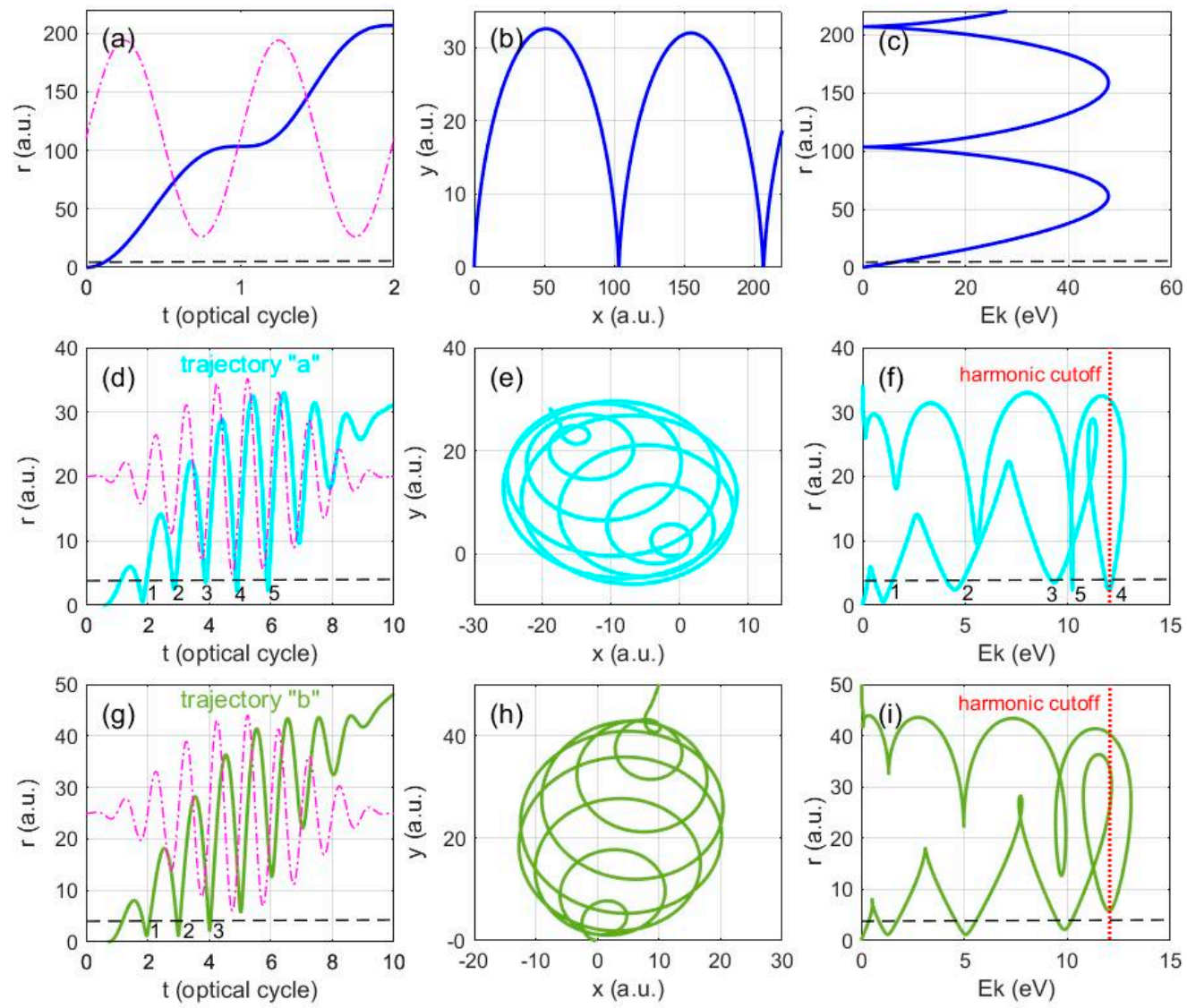

Figure 3. A typical trajectory for the laser field with a constant amplitude (a-c) and two typical trajectories for the sine-squared laser field $(\mathbf{d}-\mathbf{i})$. The first, second, and third columns present the time-dependent distance between the electron and the parent ion $r$, two-dimensional evolution of the electron displacement, and the evolution of the electron kinetic energy during the trajectory. The $x$ component of the driving field is also presented by the pink dash-dotted curves in panels $(\mathbf{a}, \mathbf{d}, \mathbf{g})$. The black dashed lines in the first and third columns denote the position $r=4.0$ a.u. The red dotted lines in the third column denote the maximum return kinetic energy of the electron, which is consistent with the harmonic cutoff.

Consistent with the TDSE simulation, the classical trajectory calculation shows that there are no efficient returns of the electron in the case of laser field with constant amplitude, but obvious electron returns in the case of laser field with a short envelope. As shown in Figure 3a for the typical trajectory of the laser field with constant amplitude, the distance between the electron and the parent ion $r$ monotonously increases. For clarity, we just show the previous-two-optical-cycle part of the trajectory, because $r$ increases very quickly to a very large value. From Figure 3b, one can see that the $x$ and $y$ components of the electron displacement can't be close to zero at the same time. Hence, the electron can't return to the parent ion. As a result, the high-order harmonics due to the recollision mechanism can't be observed in the case of the driving field with constant amplitude, as demonstrated in Figure 1a by the red dashed curve. When the driving field has a short envelope, both the $\mathrm{x}$ and $\mathrm{y}$ component of the electric field have a gradually increasing amplitude during the rising edge, i.e. a positive temporal gradient of the electric field amplitude. The greater amplitude of the electric field in the following half cycle will pull the outgoing electron towards the parent ion. Hence, $r$ presents an oscillation behavior, as shown in Figure 3d,g and Figure 4a,d for all the four typical trajectories of the sine-squared laser field. The magnitude of the oscillation is determined by the magnitude of the positive temporal gradient of the electric field amplitude. Hence, the oscillation magnitude of $r$ is similar for all the trajectories, as shown in Figure 3d,g and Figure 4a,d. This oscillation behavior of $r$ is 
quite different from that of the electron trajectory driven by the laser field with constant amplitude. It is this oscillation that makes the electron return to the parent ion possible.
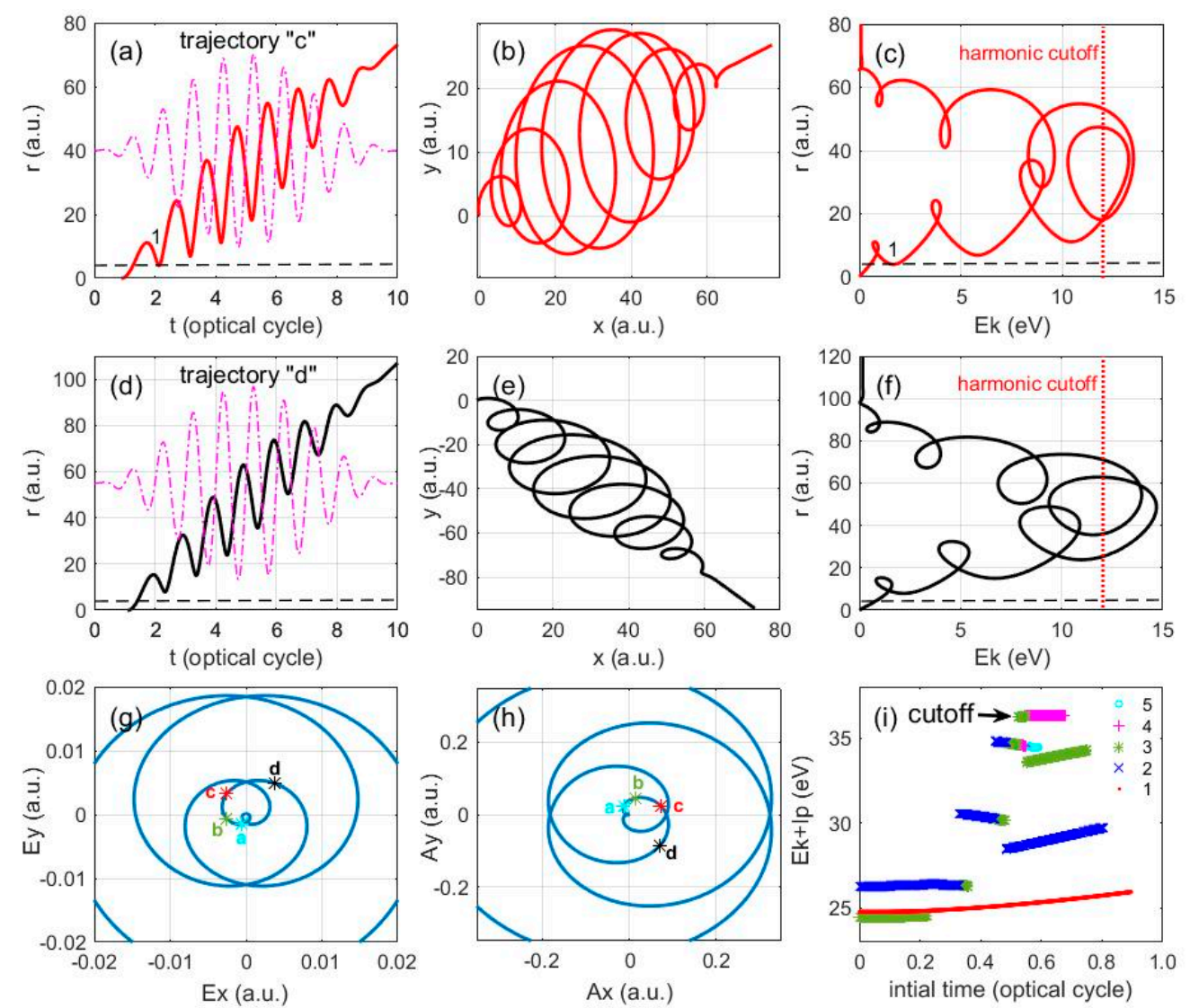

Figure 4. (a-f) Two typical trajectories for the sine-squared laser field. (g) The electric field and (h) the vector potential of the sine-squared laser field at the initial time for the four typical trajectories. (i) The return kinetic energy of the electron as a function of the initial time of the trajectory. The pink dash-dotted curves in panels $(\mathbf{a}, \mathbf{d})$ represent the $x$ component of the driving field. The red dotted lines in panels $(\mathbf{c}, \mathbf{f})$ indicate the maximum return kinetic energy of the electron, which is consistent with the harmonic cutoff. The black dashed lines in panels $(\mathbf{a}, \mathbf{c}, \mathbf{d}, \mathbf{f})$ denote the position $r=4.0$ a.u.

As shown in Figure $3 \mathrm{~d}-\mathrm{f}$ for trajectory " $\mathrm{a}$ ", the distance between the electron and the parent ion $r$ appears less than 4 a.u. for five times. Hence, the corresponding electron has five chances to return to the parent ion. The return kinetic energy of the electron reaches the maximum value of $12.01 \mathrm{eV}$ at the fourth time, as depicted in Figure $3 \mathrm{f}$ by the red dashed line. This maximum value turns out to be consistent with the harmonic cutoff around $37.19 \mathrm{eV}$. As for trajectory " $\mathrm{b}$ " shown in Figure 3g-i and trajectory " $c$ " shown in Figure $4 a-c, r$ reaches the values less than 4 a.u. for three and one times, respectively. Hence, these electrons can also return to the parent ion, but the return kinetic energy of the electron can't reach the maximum value of $12.01 \mathrm{eV}$, as shown in Figures 3i and 4c. Whereas for trajectory " $\mathrm{d}$ " shown in Figure $4 \mathrm{~d}-\mathrm{f}$, although $r$ presents the oscillation behavior, it still can't reach a value less than 4 a.u. This is because besides the oscillation, $r$ also presents a behavior that is gradually increasing at a certain velocity, as shown in the first column of Figures 3 and 4. In other words, the electron also present a behavior of drifting away from the parent ion at a certain velocity, besides the circular motion, as shown in Figure $3 \mathrm{e}, \mathrm{h}$ and Figure $4 \mathrm{~b}, \mathrm{e}$. This drifting velocity is determined by the vector potential at the initial time of the trajectory, as shown in Figure 4h. The smaller the vector potential at the initial time is, the slower $r$ increases. Because the oscillation magnitude of $r$ is similar for all the trajectories, the trajectory with a smaller drifting velocity will have more chances to reach the value less than 4 a.u. This well explains the different return times of trajectory " $a$ ", " $b$ ", 
and " $\mathrm{c}$ ". When the vector potential becomes too large, such as trajectory " $\mathrm{d}$ ", then $r$ increases too fast that it can not be less than 4 a.u. during the oscillation. Hence, the corresponding electron can't return once being away from the parent ion. In Figure $4 \mathrm{i}$, we present all the returning trajectories for the sine-squared laser field. The y axis stands for the kinetic energy at the return time plus the ionization energy of the atoms. The $\mathrm{x}$ axis stands for the initial time of the trajectory. From this figure, one can see that the returning kinetic energy of the electron generally reaches its maximum value at the third or fourth returns. This maximum value is consistent with the harmonic cutoff predicted by the TDSE simulation. From the above discussion, one can see that the oscillating behavior of $r$ is caused by the gradually increasing amplitude of the electric field, i.e. the positive temporal gradient of the electric field amplitude. This oscillation behavior makes the electron's return to the parent ion possible. Hence, the positive temporal gradient of the electric field amplitude is a fundamental key factor for the electron return and finally for the high-order harmonic generation with a circularly polarized laser field. According to the classical trajectory analysis, the larger the temporal gradient of the electric field amplitude is, the larger magnitude of the oscillation of $r$. This means that the electron will be pulled closer to the parent ion. We define the minimum value of the vector potential that leads to no return of the electron as the threshold vector potential. Then, the threshold vector potential will be larger for a laser field with a larger temporal gradient. As a result, more electron trajectories can lead to the electron's return to the parent ion. Correspondingly, the harmonic yield will be enhanced. This provides a new way of controlling the high-order harmonic generation from atoms driven by a circularly polarized laser field.

There are two methods to increase the temporal gradient of the electric field amplitude. One is increasing the intensity of the driving field, meanwhile keeping the pulse duration constant. The other one is decreasing the pulse duration but fixing the intensity of the driving field. As the harmonic yield can also be enhanced by increasing the intensity of the driving laser field, it is difficult to identify the effect of the temporal gradient of the electric field amplitude from that of the pulse intensity for the former method. Consequently, we use the latter method to test the role of the temporal gradient of the electric field amplitude in controlling the high-order harmonic generation from atoms in a circularly polarized laser field. In Figure 5a, the harmonic spectra for circularly polarized laser fields of three different pulse durations are presented. The intensity of the laser field is fixed at $1.0 \times 10^{14} \mathrm{~W} / \mathrm{cm}^{2}$. The three different pulse durations are 10, 50, and 90 optical cycles, respectively. For a comparison, the harmonic spectra for linearly polarized laser fields are also presented in Figure $5 b$. As shown in Figure $5 \mathrm{a}$ for circularly polarized laser field, the harmonic yield increases with decreasing pulse duration. Whereas, in the case of linearly polarized laser field, the harmonic yield decreases with decreasing pulse duration. For clarity, we used Equation (7) to calculate the integrated harmonic yield near the harmonic cutoff from $27.19 \mathrm{eV}$ to $37.19 \mathrm{eV}$ and more pulse durations are considered. The result is shown in Figure 5c. One can clearly see that the integrated harmonic yield increases with shortening the pulse duration. Whereas in the case of a linearly polarized laser field, the integrated harmonic yield near the cutoff from $56.67 \mathrm{eV}$ to $66.67 \mathrm{eV}$ decreases with shortening the pulse duration, as shown in Figure $5 \mathrm{~d}$. This is because in the case of linearly polarized laser field, the electron return to the parent ion happens every half cycle, independent of the temporal gradient of the electric field amplitude. The longer the pulse duration is, the more electron returns there will be. The total harmonic yield is determined by adding the contributions of all the electron returns happening during the whole pulse duration. Hence, the harmonic yield increases with increasing the pulse duration, as shown in Figure $5 b$,d. Hence, it is confirmed that in the case of circularly polarized laser field, the larger the temporal gradient of the electric field amplitude is, the more trajectories can lead to the electron return. As a result, the harmonic yield increases. 

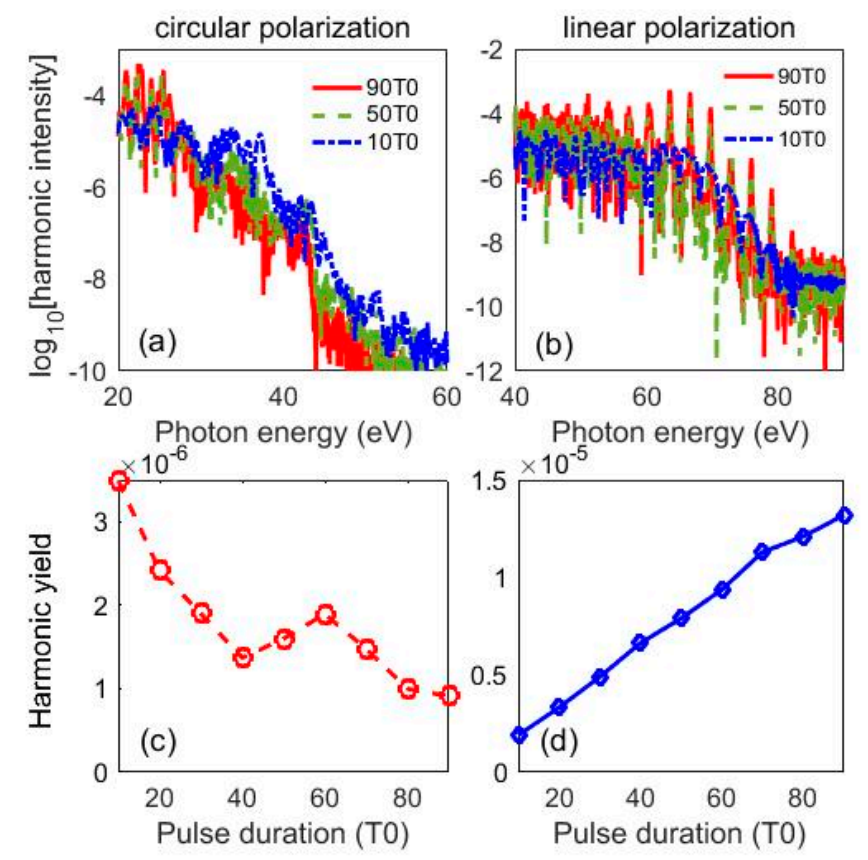

Figure 5. (a-b) Pulse-duration dependence of high-order harmonic spectrum for circularly polarized (a) and linearly polarized (b) sine-squared laser fields. The intensity and wavelength of the laser fields are fixed at $1.0 \times 10^{14} \mathrm{~W} / \mathrm{cm}^{2}$ and $800 \mathrm{~nm}$, respectively. (c-d) Pulse-duration dependence of integrated harmonic yields for circularly polarized laser field from $27.19 \mathrm{eV}$ to $37.19 \mathrm{eV}$ (c) and linearly polarized laser fields from $56.67 \mathrm{eV}$ to $66.67 \mathrm{eV}$ (d).

\section{Conclusions}

In conclusion, the influence of the waveform of circularly polarized laser field on high-order harmonic $(\mathrm{HH})$ generation from atoms is investigated by solving the time-dependent Schrödinger equation (TDSE) and the classical trajectory analysis beyond the transverse-velocity-compensation mechanism. It is found that the $\mathrm{HH}$ emissions due to the electron recollision can be switched on or off by modulating the driving laser field with a finite envelope. By a detailed analysis of the classical trajectories of the ionized electron without an initial transverse velocity, we demonstrate that due to the positive temporal gradient, the amplitude of the driving field in the following half cycle is larger than that of the previous half cycle. The larger amplitude of the electric field can pull the previously outgoing electron back to the vicinity of the parent ion. Hence, the electron has the chance to recombine with the parent ion. Moreover, the greater the temporal gradient of the laser field amplitude, the more electron trajectories can return to the vicinity of the parent ion. Correspondingly, the more electrons can recombine with the parent ion and emit the high-order harmonics. As a result, the greater harmonic yield is obtained. This is confirmed by the dependence of the harmonic yield on the pulse duration of the circularly polarized driving field. In addition, the maximum kinetic energy of the return electron, which agrees well with the harmonic cutoff position, is generally gained after multiple revisits. Our results provide a new way of controlling the $\mathrm{HH}$ emission in a circularly polarized laser field.

Author Contributions: Conceptualization, Q.L.; Data curation, M.Q.; Formal analysis, M.Q.; Funding acquisition, M.Q.; Investigation, M.Q., Y.Z. and X.Z.; Supervision, Q.L.; Writing - original draft, M.Q.; Writing - review \& editing, M.Q., Y.Z., X.Z. and Q.L.

Acknowledgments: This work was supported by the National Natural Science Foundation of China under Grants (No.11604248, No. 11674257, No. 11747041), Undergraduate Headmaster Foundation of Wuhan Institute of Technology (No. 2018131), National College Students' innovation and entrepreneurship training program (No. 201810490023), the Program for Distinguished Middle-aged and Young Innovative Research Team in Higher Education of Hubei, China (No. T201806), and the Natural Science Foundation of Hubei Province under Grants No. 2017CFB150.

Conflicts of Interest: The authors declare no conflict of interest. 


\section{References}

1. Itatani, J.; Levesque, J.; Zeidler, D.; Niikura, H.; Pepin, H.; Kieffer, J.C.; Corkum, P.B.; Villeneuve, D.M. Tomographic imaging of molecular orbitals. Nature 2004, 432, 867-871. [CrossRef] [PubMed]

2. Lan, P.; Ruhmann, M.; He, L.; Zhai, C.; Wang, F.; Zhu, X.; Zhang, Q.; Zhou, Y.; Li, M.; Lein, M.; et al. Attosecond Probing of Nuclear Dynamics with Trajectory-Resolved High-Harmonic Spectroscopy. Phys. Rev. Lett. 2017, 119, 033201. [CrossRef] [PubMed]

3. He, L.; Zhang, Q.; Lan, P.; Cao, W.; Zhu, X.; Zhai, C.; Wang, F.; Shi, W.; Li, M.; Bian, X.; et al. Monitoring ultrafast vibrational dynamics of isotopic molecules with frequency modulation of high-order harmonics. Nat. Commun. 2018, 9, 1108. [CrossRef] [PubMed]

4. Qin, M.; Zhu, X. Molecular orbital imaging for partially aligned molecules. Opt. Laser Technol. 2017, 87, 79-86. [CrossRef]

5. Wei, P.; Qin, M.; Yuan, X.; Liu, C.; Li, R.; Zeng, Z.; Lu, P.; Dorfman, K.; Ye, W.; Yao, B.; et al. Probing electron-atom collision dynamics in gas plasma by high-order harmonic spectroscopy. Opt. Lett. 2018, 43, 1970-1973. [CrossRef] [PubMed]

6. Zhou, Y.; Tolstikhin, O.I.; Morishita, T. Near-forward rescattering photoelectron holography in strong-field ionization: Extraction of the phase of the scattering amplitude. Phys. Rev. Lett. 2016, 116, 173001. [CrossRef] [PubMed]

7. He, M.; Li, Y.; Zhou, Y.; Li, M.; Cao, W.; Lu, P. Direct visualization of valence electron motion using strong-field photoelectron holography. Phys. Rev. Lett. 2017, 120, 133204. [CrossRef] [PubMed]

8. Tong, A.; Zhou, Y.; Lu, P. Bifurcation of ion momentum distributions in sequential double ionization by elliptically polarized laser pulses. Opt. Quant. Electron. 2017, 49, 77. [CrossRef]

9. Tan, J.; Li, Y.; Zhou, Y.; He, M.; Chen, Y.; Li, M.; Lu, P. Identifying the contributions of multiple-returning recollision orbits in strong-field above-threshold ionization. Opt. Quant. Electron. 2018, 50, 57. [CrossRef]

10. Ma, X.; Li, M.; Zhou, Y.; Lu, P. Nonsequential double ionization of Xe by mid-infrared laser pulses. Opt. Quant. Electron. 2017, 49, 170. [CrossRef]

11. Niikura, H.; Légaré, F.; Hasbani, R.; Bandrauk, A.D.; Ivanov, M.Y.; Villeneuve, D.M.; Corkum, P.B. Sub-laser-cycle electron pulses for probing molecular dynamics. Nature 2002, 417, 917. [CrossRef] [PubMed]

12. Jia, G.; Huang, X.; Bian, X. Nonadiabatic redshift in high-order harmonic generation from solids. Opt. Express 2017, 25, 23654-23662. [CrossRef] [PubMed]

13. Chen, Y.; Zhou, Y.; Li, Y.; Li, M.; Lan, P.; Lu, P. Rabi oscillation in few-photon double ionization through doubly excited states. Phys. Rev. A 2018, 97, 013428. [CrossRef]

14. Kfir, O.; Grychtol, P.; Turgut, E.; Knut, R.; Zusin, D.; Popmintchev, D.; Popmintchev, T.; Nembach, H.; Shaw, J.M.; Fleischer, A.; et al. Generation of bright phase-matched circularly-polarized extreme ultraviolet high harmonics. Nat. Photonics 2015, 9, 99-105. [CrossRef]

15. Yuan, K. Symmetry in circularly polarized molecular high-order harmonic generation with intense bicircular laser pulses. Phys. Rev. A 2018, 97, 023408. [CrossRef]

16. Lambert, G.; Vodungbo, B.; Gautier, J.; Mahieu, B.; Malka, V.; Sebban, S.; Zeitoun, P.; Luning, J.; Perron, J.; Andreev, A.; et al. Towards enabling femtosecond helicity-dependent spectroscopy with high harmonic sources. Nat. Commun. 2015, 6, 6167. [CrossRef]

17. Jimenez-Galan, A.; Dixit, G.; Patchkovskii, S.; Smirnova, O.; Morales, F.; Ivanov, M. Attosecond recorder of the polarization state of light. Nat. Commun. 2018, 9, 850. [CrossRef]

18. Corkum, P.B. Plasma perspective on strong-field multiphoton ionization. Phys. Rev. Lett. 1993, 71, $1994-1997$. [CrossRef]

19. Lewenstein, M.; Balcou, P.; Ivanov, M.Y.; L'Huillier, A.; Corkum, P.B. Theory of high-harmonic generation by low-frequency laser fields. Phys. Rev. A 1994, 49, 2117-2132. [CrossRef]

20. Budil, K.S.; Salières, P.; L'Huillier, A.; Ditmire, T.; Perry, M.D. Influence of ellipticity on harmonic generation. Phys. Rev. A 1993, 48, R3437. [CrossRef]

21. Corkum, P.B.; Burnett, N.H.; Ivanov, M.Y. Subfemtosecond pulses. Opt. Lett. 1994, 19, 1870-1872. [CrossRef] [PubMed]

22. Dietrich, P.; Burnett, N.H.; Ivanov, M.; Corkum, P.B. High-harmonic generation and correlated two-electron multiphoton ionization with elliptically polarized light. Phys. Rev. A 1994, 50, R3585-R3588. [CrossRef] [PubMed] 
23. Antoine, P.; L'huillier, A.; Lewenstein, M. Theory of high-order harmonic generation by an elliptically polarized laser field. Phys. Rev. A 1996, 53, 1725. [CrossRef]

24. Weihe, F.A.; Bucksbaum, P.H. Measurement of the polarization state of high harmonics generated in gases. J. Opt. Soc. Am. B 1996, 13, 157-161. [CrossRef]

25. Sansone, G.; Benedetti, E.; Calegari, F.; Vozzi, C.; Avaldi, L.; Flammini, R.; Poletto, L.; Villoresi, P.; Altucci, C.; Velotta, R.; et al. Isolated single-cycle attosecond pulses. Science 2006, 314, 443-446. [CrossRef] [PubMed]

26. Khan, S.D.; Cheng, Y.; Möller, M.; Zhao, K.; Zhao, B.; Chini, M.; Paulus, G.G.; Chang, Z. Ellipticity dependence of $400 \mathrm{~nm}$-driven high harmonic generation. Appl. Phys. Lett. 2011, 99, 161106. [CrossRef]

27. Möller, M.; Cheng, Y.; Khan, S.D.; Zhao, B.; Zhao, K.; Chini, M.; Paulus, G.G.; Chang, Z. Dependence of high-order-harmonic-generation yield on driving-laser ellipticity. Phys. Rev. A 2012, 86, 011401. [CrossRef]

28. Li, Y.; Zhu, X.; Zhang, Q.; Qin, M.; Lu, P. Quantum-orbit analysis for yield and ellipticity of high order harmonic generation with elliptically polarized laser field. Opt. Express 2013, 21, 4896-4907. [CrossRef] [PubMed]

29. Zhu, X.; Liu, X.; Li, Y.; Qin, M.; Zhang, Q.; Lan, P.; Lu, P. Molecular high-order-harmonic generation due to the recollision mechanism by a circularly polarized laser pulse. Phys. Rev. A 2015, 91, 043418. [CrossRef]

30. Wang, X.; Eberly, J.H. Elliptical polarization and probability of double ionization. Phys. Rev. Lett. 2010, 105, 083001. [CrossRef]

31. Feit, M.D.; Fleck, J.A.; Steiger, A. Solution of the Schrödinger equation by a spectral method. J. Comput. Phys. 1982, 47, 412-433. [CrossRef]

32. Burnett, K.; Reed, V.C.; Cooper, J.; Knight, P.L. Calculation of the background emitted during high-harmonic generation. Phys. Rev. A 1992, 45, 3347. [CrossRef] [PubMed]

33. Liao, Q.; Winney, A.H.; Lee, S.K.; Lin, Y.F.; Adhikari, P.; Li, W. Coulomb-repulsion-assisted double ionization from doubly excited states of argon. Phys. Rev. A 2017, 96, 023401. [CrossRef]

(C) 2019 by the authors. Licensee MDPI, Basel, Switzerland. This article is an open access article distributed under the terms and conditions of the Creative Commons Attribution (CC BY) license (http:/ / creativecommons.org/licenses/by/4.0/). 